ARTICLES

\title{
RELATIVITÉ D'ÉCHELLE STRUCTURE DE LA THÉORIE
}

\author{
Laurent NotTALE
}

Résumé: La théorie de la relativité d'échelle développe les conséquences de l'abandon de l'hypothèse de différentiabilité des coordonnées spatio-temporelles. La première est le caractère fractal, c'est-à-dire explicitement dépendant des résolutions, qu'acquiert l'espace-temps. On redéfinit alors les résolutions comme caractérisant l'état d'échelle du référentiel, puis on postule un principe de relativité d'échelle, suivant lequel les lois de la nature doivent être valides quel que soit cet état. Il s'agit ainsi de construire une extension des théories existantes de la relativité, qui s'appliquaient jusqu'à maintenant aux changements d'état de position, d'orientation et de mouvement. Par conséquent, la structure de la théorie suit un cheminement parallèle aux différents niveaux de la théorie relativiste (galiléenne, einsteinienne restreinte puis générale), auxquels s'ajoutent les effets du couplage entre échelle et mouvement.

Mots-CLÉs : relativité, échelles, dilatations, espace-temps, fractales, équations différentielles.

ABSTRACT: The theory of scale relativity studies the consequences of giving up the hypothesis of the differentiability of space-time coordinates. The first consequence is that space-time acquires a fractal geometry, i.e., it becomes explicitly resolution dependent. One redefines resolutions as characterizing the state of scale of the reference system, then one postulates a principle of scale relativity, according to which the laws of nature should be valid whatever this state. The question is to construct an extension of presently existing theories of relativity, that applied up to now to changes of state of position, orientation and motion. As a consequence the structure of the theory follows a path parallel to the various levels of the standard relativistic theory (Galilean, Einsteinian special then general), to which one adds the effects of coupling between scale and motion.

KEYWORDS : relativity, scales, dilations, space-time, fractals, differential equations.

Revue de synthèse : $4^{\mathrm{e}} \mathrm{S} . \mathrm{n}^{\mathrm{o}}$ 1, janv.-mars 2001, p. 11-25. 
Zusammenfassung: In der Theorie der Skalen-Relativität geht es um die Konsequenzen, die sich ergeben, wenn man auf die Annahme verzichtet, daß die Koordinaten der Raumzeit differenzierbar sind. Die erste Konsequenz ist die fraktale Geometrie der Raumzeit, d.h. sie wird explizit abhängig von der Auflösung. Dabei werden Auflösungen als skalare Zustandsmerkmale des Bezugssystems definiert, und anschließend wird ein skalares Relativitätsprinzip postuliert, das besagt, daß die Naturgesetze unabhängig vom jeweiligen Zustand gelten sollen. Es geht somit um die Erweiterung der zur Zeit bestehenden Relativitätstheorien, die bis jetzt nur für Zustandsänderungen der Lage, der Richtung und der Bewegung gelten. Folglich entwickelt sich die Struktur der Theorie parallel zu den verschiedenen Ebenen der bisherigen Relativitätstheorie (Galileisches Relativitätsprinzip, Spezielle und Allgemeine Relativitätstheorie Einsteins). Hinzu kommen die Effekte der Kopplung von Maßstab und Bewegung.

STICHWÖRTER: Relativität, Maßstäbe, Ausdehnungen, Raumzeit, Fraktale, Differentialgleichungen.

RIASSUNTO : La teoria della relatività di scala sviluppa le conseguenze dell'abbandono dell'ipotesi della differenziabilità delle coordinate spazio-temporali. La prima è il carattere frattale, ovvero il carattere esplicitamente dipendente dalle risoluzioni che acquisisce lo spazio-tempo. Si ridefiniscono quindi le risoluzioni comme caratterizzanti dello stato di scala del referenziale, inoltre si postula un principio di relatività di scala, secondo il quale le leggi della natura devono essere valide qualunque sia lo stato. Si tratta allora di costruire un'estensione delle teorie della relatività esistenti, che si applicavano fin'ora ai cambiamenti di stato, di posizione, d'orientamento e di movimento. Di conseguenza, la struttura della teoria segue un cammino parallelo ai diversi livelli della teoria relativista (gallileana, einsteiniana ristretta e poi generale), alla quale si aggiungono gli effetti dell'associazione tra scala e movimento.

PAROLE CHIAVE : relatività, scale, dilatazioni, spazio-tempo, frattali, equazioni differenziali.

Laurent NotTale, né en 1952, est directeur de recherche au Centre national de la recherche scientifique, à l'observatoire de Paris-Meudon. Il est spécialiste d'astrophysique et de physique théorique.

Adresse : DAEC/CNRS, Observatoire de Meudon, F-92195 Meudon Cedex.

Courrier électronique : laurent.nottale@obspm.fr 
La théorie de la relativité d'échelle consiste à appliquer le principe de relativité aux transformations d'échelle (en particulier aux transformations des résolutions spatio-temporelles). Dans la formulation d'Einstein ${ }^{1}$, le principe de relativité consiste à exiger que les lois de la nature soient valides dans tout système de coordonnées, quel que soit son état. Depuis Galilée, ce principe avait été appliqué aux états de position (origine et orientation des axes) et de mouvement du système de coordonnées (vitesse, accélération), états qui ont la propriété de n'être jamais définissables de manière absolue, mais seulement de manière relative entre deux repères. Il en est de même en ce qui concerne les problèmes d'échelle. L'échelle d'un système ne peut être définie que par rapport à un autre système (seuls des rapports d'échelle ont un sens, jamais une échelle absolue), et possède donc bien la propriété fondamentale de relativité. Dans la nouvelle approche, on réinterprète les résolutions, non plus seulement comme propriété de l'appareil de mesure et/ou du système mesuré, mais comme propriété intrinsèque à l'espace-temps, caractérisant l'état d'échelle du référentiel au même titre que les vitesses caractérisent son état de mouvement. Le principe de relativité d'échelle consiste alors à demander que les lois fondamentales de la nature s'appliquent quel que soit l'état d'échelle du système de coordonnées.

Nous allons, dans le présent article, tenter d'expliciter la structure des développements (déjà réalisés, en cours et en projet) d'une telle approche théorique $^{2}$. Il s'agit d'un programme à long terme, qui a déjà donné des résultats partiels (en particulier, certaines prédictions théoriques ont maintenant reçu des confirmations observationnelles, comme celle de structures gravitationnelles universelles depuis l'échelle des systèmes planétaires jusqu'à des échelles extragalactiques ou encore la valeur de la constante cosmologique), mais qui reste essentiellement ouvert : en effet, de nombreuses voies de recherche impliquées par ce programme n'ont encore été qu'effleurées. Au cours et à l'occasion de cette description, nous essaierons de montrer en quoi le concept de relativité d'échelle peut nous permettre de réviser celui d'espace-temps.

1. Einstein, 1916.

2. Pour plus de détails, voir Nottale, 1993, 1996 et 1998. 


\section{1. — ABANDON DE L'HYPOTHÈSE DE DIFFÉRENTIABILITÉ}

Si l'on analyse l'état de la physique fondée sur le principe de relativité jusqu'à Einstein, on constate que c'est l'ensemble de la physique classique, y compris la théorie de la gravitation à travers la relativité généralisée du mouvement, qui est fondée sur lui. La physique quantique, bien que compatible avec les relativités galiléenne et restreinte du mouvement, semble y échapper en ce qui concerne ses fondations. On peut alors se demander si une nouvelle généralisation de la relativité reste possible, qui inclurait les effets quantiques (ou au moins certains d'entre eux) dans ses conséquences. Or, généraliser la relativité, c'est généraliser les transformations envisageables entre systèmes de coordonnées, c'est donc généraliser la définition de ce que sont les systèmes de référence possibles, et c'est finalement généraliser les concepts d'espace et d'espace-temps. La relativité générale d'Einstein repose sur l'hypothèse que l'espace-temps est riemannien, c'est-à-dire descriptible par une variété au moins deux fois différentiable : autrement dit, on peut définir un continuum d'événements spatio-temporels, puis des vitesses qui sont leur dérivées, puis des accélérations par une nouvelle dérivation. Dans ce cadre, les équations d'Einstein sont les plus générales des équations les plus simples qui soient covariantes dans des transformations de coordonnées deux fois différentiables.

De même que le passage de la relativité restreinte à la relativité généralisée est permis par un abandon d'hypothèse restrictive (celle de la platitude de l'espace-temps à travers la prise en considération d'espaces-temps courbes), une nouvelle ouverture est alors possible par l'abandon de l'hypothèse de différentiabilité. Il s'agira de décrire un continuum spatiotemporel qui ne soit plus forcément partout différentiable.

\section{2. - CONSÉQueNCE : DÉPENDANCE EXPLICITE (DIVERGENCE) DES COORDONNÉES EN FONCTION DES RÉSOLUTIONS, CARACTÈRE FRACTAL DE L'ESPACE-TEMPS}

La deuxième étape de la construction consiste à récupérer un outil physico-mathématique qui pourrait sembler avoir été perdu dans une telle généralisation. L'outil essentiel de la physique, depuis Galilée, Leibniz et Newton, ce sont les équations différentielles. Abandonner l'hypothèse de la différentiabilité de l'espace-temps, donc de celle des systèmes de coordon- 
nées et des transformations entre ces systèmes, n'est-ce pas abandonner les équations différentielles?

Ce problème crucial peut en fait être contourné grâce à l'intervention d'un concept nouveau en physique de l'espace-temps : celui de géométries fractales. Par leur biais, on peut traiter de non-différentiabilité à l'aide d'équations différentielles.

Cette possibilité résulte du théorème suivant ${ }^{3}$, lui-même conséquence d'un théorème de Henri Lebesgue. On démontre qu'une courbe continue mais presque partout non dérivable possède une longueur dépendant explicitement de la résolution à laquelle on la considère, et tendant vers l'infini quand l'intervalle de résolution tend vers zéro. Autrement dit, une telle courbe est fractale au sens général donné par Benoît Mandelbrot ${ }^{4}$ à ce terme. Appliqué à un système de coordonnées d'un espace-temps nondifférentiable, ce théorème implique une géométrie fractale pour cet espace-temps ${ }^{5}$, aussi bien que pour le référentiel. De plus, c'est la dépendance en fonction des résolutions elle-même qui résout le problème posé. Considérons en effet la définition de la dérivée, appliquée par exemple à la variable de position :

$$
v(t)=\lim _{d t \rightarrow 0}[x(t+d t)-x(t)] / d t .
$$

La non-différentiabilité est la non-existence de cette limite. Celle-ci étant, de toutes façons, physiquement inatteignable (l'atteindre effectivement nécessiterait une énergie infinie d'après la relation temps-énergie de Heisenberg), on redéfinit $v$ comme $v(t, d t)$, fonction du temps $t$ et de l'élément différentiel $d t$ identifié à un intervalle de résolution. La question n'est alors plus la description de ce qui se passe à la limite, mais bien du comportement de cette fonction au cours de zooms successifs sur l'intervalle $d t$.

3. - PRINCIPE DE RELATIVITÉ ET DE COVARIANCE D’ÉCHELLE PORTANT SUR LES TRANSFORMATIONS (CONTRACTIONS ET DILATATIONS) DES RÉSOLUTIONS SPATIO-TEMPORELLES

Il s'agit ainsi de compléter notre description actuelle, qui est faite en terme d'espace (de positions), d'espace-temps ou d'espace des phases, par un espace des échelles. De même que les vitesses caractérisent l'état de mouvement du système de coordonnées, on considérera que les résolutions caractérisent son état d'échelle. La nature relative des intervalles de résolu-

3. Nottale, 1993, 1996 et 1997a.

4. Mandelbrot, 1975 et 1982.

5. Nottale et Schneider, 1984; Ord, 1983; El Naschie, 1995. 
tion temporel et spatial s'impose d'elle-même : seul un rapport d'intervalles de longueur ou de temps peut être défini, jamais leur valeur absolue, comme en témoigne la nécessité d'avoir toujours recours à des unités. Cette relativité des échelles permet de postuler un principe de relativité d'échelle, selon lequel les lois fondamentales de la nature doivent s'appliquer quel que soit l'état d'échelle du système de référence. Dans ce cadre, on appelle covariance d'échelle l'invariance de forme des équations de la physique sous les transformations des résolutions spatio-temporelles. Il convient de prendre garde au fait que cette expression a été introduite par d'autres auteurs en un sens légèrement différent, comme généralisation de l'invariance d'échelle).

\section{4. - RECHERCHE DES ÉQUATIONS DIFFÉRENTIELLES EN ÉCHELLE SATISFAISANT AU PRINCIPE DE RELATIVITÉ D'ÉCHELLE}

Les différentes grandeurs physiques, au premier rang desquelles les coordonnées et les vitesses elles-mêmes, sont maintenant considérées comme dépendant explicitement des résolutions. Quelle est la nature de cette dépendance ? On répondra à cette question en considérant qu'elle doit satisfaire à des équations différentielles dans l'espace des échelles. Autrement dit, on considérera un zoom infinitésimal $\ln \varepsilon \rightarrow \ln \varepsilon+d \ln \varepsilon$, et l'on cherchera à définir comment la grandeur considérée s'est elle-même transformée au cours de ce zoom, en imposant que cette transformation doit satisfaire au principe de relativité d'échelle.

\subsection{Identification des lois de puissance fractales ordinaires (à dimension} fractale constante) comme relativité galiléenne d'échelle

\subsubsection{Groupe de Galilée des transformations d'échelle}

Les lois de puissance rencontrées dans les comportements fractals autosimilaires peuvent être identifiées comme les plus simples des lois recherchées : elles correspondent en ce qui concerne les échelles à ce qu'est l'inertie du point de vue du mouvement. On peut s'en assurer facilement en appliquant une transformation de résolution à une longueur mesurée sur une courbe fractale, (qui décrira en particulier une coordonnée dans un système de référence fractal) :

$$
L=L_{0}\left(\lambda_{0} / \varepsilon\right)^{\delta},
$$


où $D=\delta+1$ est la dimension fractale, supposée constante à ce niveau d'analyse. Sous une transformation d'échelle $\varepsilon \rightarrow \varepsilon^{\prime}$, on obtient :

$$
\begin{gathered}
\ln L^{\prime}=\ln L+\delta \ln \left(\varepsilon / \varepsilon^{\prime}\right), \\
\delta^{\prime}=\delta,
\end{gathered}
$$

où l'on reconnaît la structure mathématique du groupe de transformation de Galilée entre systèmes inertiels : la substitution (mouvement $\rightarrow$ échelle) se traduit par les correspondances $x \rightarrow \ln L, t \rightarrow \delta, v \rightarrow \ln \left(\varepsilon / \varepsilon^{\prime}\right)$. Noter la manifestation de la relativité des résolutions du point de vue mathématique: elles n'interviennent que par leurs rapports et l'échelle arbitraire $\lambda_{0}$ a disparu dans la relation (3a).

En accord avec l'analyse précédente du statut des résolutions en physique, la dimension d'échelle joue pour les échelles le rôle joué par le temps en ce qui concerne le mouvement, et le logarithme du rapport des résolutions celui de la vitesse. La loi de composition des dilatations écrite sous forme logarithmique confirme cette identification avec le groupe de Galilée :

$$
\ln \left(\varepsilon^{\prime \prime} / \varepsilon\right)=\ln \left(\varepsilon^{\prime \prime} / \varepsilon^{\prime}\right)+\ln \left(\varepsilon^{\prime} / \varepsilon\right)
$$

formellement identique à la composition galiléenne des vitesses, $w=u+v$.

\subsubsection{Brisure spontanée de l'invariance d'échelle}

L'expression (2) est invariante d'échelle. Cette invariance est spontanément brisée par l'existence du déplacement et du mouvement. Changeons, en effet, l'origine du système de coordonnées. Nous obtenons

$$
L=L_{0}\left(\lambda_{0} / \varepsilon\right)^{\delta}+L_{1}=L_{1}\left(1+\left(\lambda_{1} / \varepsilon\right)^{\delta}\right),
$$

où $\lambda_{1}=\lambda_{0}\left(L_{0} / L_{1}\right)^{1 / \delta}$. Alors que l'échelle $\lambda_{0}$ restait arbitraire, l'échelle $\lambda_{1}$ (qui reste relative du point de vue de l'espace-temps) prend le sens d'une échelle de brisure de symétrie d'échelle (autrement dit, de transition fractal-non fractal dans l'espace des échelles). En effet, il est aisé de constater que pour $\varepsilon>\lambda_{1}, L \approx L_{1}$ ne dépend plus de la résolution, tandis que pour $\varepsilon<<\lambda_{1}$, on récupère la dépendance en échelle donnée par (2), asymptotiquement invariante d'échelle.

\subsubsection{Plus simple équation différentielle en échelle}

Or ce comportement (5), qui satisfait donc au double principe de relativité du mouvement et d'échelle, est précisément obtenu comme solution de la plus simple des équations différentielles en échelle qu'on puisse écrire (équation du premier ordre, ne dépendant que de $L$ lui-même, cette dépendance étant développable en série de Taylor) :

$$
d L / d \ln \varepsilon=\beta(L)=a+b L+\ldots
$$


La solution de (6) est effectivement donnée par l'expression (5), avec $\delta=-b, L_{1}=-a / b$, sachant que $\lambda_{1}$ est une constante d'intégration.

\subsubsection{Espace-temps-djinn à cinq dimensions}

Notons pour finir le renversement essentiel de point de vue impliqué par la présente approche en comparaison avec la description usuelle d'objets fractals. Ce renversement est parallèle, en ce qui concerne les échelles, à celui qui fut opéré pour les lois du mouvement dans le passage de lois « aristotéliciennes » aux lois galiléennes.

Du point de vue aristotélicien, le temps est la mesure du mouvement : il se définit donc à partir de l'espace et de la vitesse. De même, la dimension fractale est définie par Mandelbrot à partir de la mesure sur l'objet fractal (par exemple, la longueur d'une courbe) et de la résolution :

$$
\ll t=x / v » \quad \leftrightarrow \quad \delta=d \ln L / d \ln \varepsilon
$$

Avec Galilée, le temps devient une variable primaire et la vitesse se déduit de l'espace et du temps, qui se retrouvent sur un même plan, sous forme d'un espace-temps (qui reste cependant dégénéré, car la vitesse limite $c$ y est implicitement infinie). Le caractère vectoriel de la vitesse et son aspect local (finalement mis en œuvre par sa définition comme dérivée de la position par rapport au temps) s'affirment. Le même renversement peut s'appliquer aux échelles. La dimension d'échelle $\delta$ devient elle-même variable primaire, traitée sur le même plan que l'espace et le temps, et les résolutions sont alors définies comme dérivées à partir de la coordonnée fractale et de $\delta$. Ce sens nouveau et fondamental donné à la dimension d'échelle rend nécessaire de lui attribuer un nouveau nom. Nous l'appellerons « djinn » dans la suite (dans des articles précédents, nous avions proposé le terme «zoom », mais celui-ci est déjà connoté et s'applique naturellement à la «vitesse relative d'échelle», $\left.\ln \left(\varepsilon^{\prime} / \varepsilon\right)\right)$. Il s'agit donc de travailler dans un espace-temps-djinn à cinq dimensions. Le caractère vectoriel des zooms est alors apparent, car les quatre résolutions spatiotemporelles peuvent maintenant se définir à partir des quatre coordonnées d'espace-temps et du djinn :

$$
v^{i}=d x^{i} / d t \quad \leftrightarrow \quad-\ln \varepsilon^{u}=d \ln L^{\mu} / \mathrm{d} \delta
$$

On pourrait objecter que du point de vue des mesures, c'est à $L$ et $\varepsilon$ que l'on a accès et que le djinn s'en déduit. Mais on remarquera qu'il en est de même de la variable temporelle, qui reste toujours mesurée de manière indirecte. 
Un dernier avantage de ce renversement sera apparent dans la suite, dans les tentatives de construction d'une relativité d'échelle généralisée. Il permet, en effet, la définition d'un concept nouveau, celui de l'accélération d'échelle, $\Gamma^{\mu}=\mathrm{d}^{2} \ln L^{\mu} / \mathrm{d} \delta^{2}$, nécessaire au passage à des lois d'échelle non linéaires et à une «dynamique» d'échelle.

L'introduction de ce concept permet de renforcer encore l'identification des fractals à dimension constante à une «inertie d'échelle». En effet, l'équation «du vide» en échelle doit s'écrire (à une dimension pour simplifier l'écriture) :

Elle s'intègre comme

$$
\Gamma=\mathrm{d}^{2} \ln L / d \delta^{2}=0 .
$$

$$
d \ln L / d \delta=-\ln \varepsilon=\text { constante. }
$$

La constance de la résolution signifie ici son indépendance en fonction du djinn $\delta$. La solution prend finalement la forme attendue (2), $L=L_{0}\left(\lambda_{0} / \varepsilon\right)^{\delta}$.

4.2. Généralisation: relativité restreinte d'échelle, lois de dilatation loglorentziennes, nouveau statut de l'échelle de Planck, covariance d'échelle

C'est avec la relativité restreinte d'échelle que le concept d'espacetemps-djinn prend tout son sens. Cependant, celle-ci n'a été développée, jusqu'à maintenant, qu'à deux dimensions, une dimension d'espace-temps et le djinn. Un traitement complet à cinq dimensions reste à faire.

La remarque de la section précédente suivant laquelle les lois fractales standard (à dimension fractale constante) ont la structure du groupe de Galilée implique aussitôt une possibilité de généralisation de ces lois. En effet, on sait depuis les travaux de Henri Poincaré ${ }^{6}$ que, en ce qui concerne le mouvement, ce groupe n'est qu'un cas très particulier et dégénéré du groupe de Lorentz. Or, on peut montrer ${ }^{7}$ qu'à deux dimensions, sur les seules hypothèses que la loi de transformation recherchée soit linéaire, interne et invariante par réflexion (hypothèses déductibles du principe de relativité restreinte), on trouve comme seule solution physiquement admissible le groupe de Lorentz : celui-ci correspond à une métrique minkowskienne (l'autre solution possible est la métrique euclidienne, qui conduit à des absurdités physiques).

Désignons par $\mathcal{L}$ la différence $\mathcal{L}=L-L_{1}$, qui décrit le comportement fractal asymptotique (ce qui permet de prendre en compte automatiquement la transition fractal-non fractal $\lambda$ ). En fonction du rapport de dilatation $\rho$ dans une transformation entre échelles de résolutions $\varepsilon \rightarrow \varepsilon^{\prime}$, la nouvelle transformation d'échelle log-lorentzienne s'écrit :

6. Poincaré, 1905.

7. NotTale, 1992. 


$$
\begin{aligned}
& \ln \left(\mathcal{L}^{\prime} / \mathcal{L}_{0}\right)=\frac{\ln \left(\mathcal{L} / \mathcal{L}_{0}\right)+\delta \ln \rho}{\sqrt{1-\ln ^{2} \rho / \ln ^{2}(\lambda / \Lambda)}}, \\
& \delta^{\prime}=\frac{\delta+\ln \rho \ln \left(\mathcal{L} / \mathcal{L}_{0}\right) / \ln ^{2}(\lambda / \Lambda)}{\sqrt{1-\ln ^{2} \rho / \ln ^{2}(\lambda / \Lambda)}} .
\end{aligned}
$$

La loi de composition des dilatations prend la forme :

$$
\ln \frac{\varepsilon^{\prime}}{\lambda}=\frac{\ln (\varepsilon / \lambda)+\ln \rho}{1+\frac{\ln \rho \ln (\varepsilon / \lambda)}{\ln ^{2}(\lambda / \Lambda)}} .
$$

Précisons que ces lois ne sont valables qu'en deçà de l'échelle de transition $\lambda$ (respectivement, au-delà de cette échelle de transition dans l'application de cette loi vers les très grandes échelles). Comme on peut le constater sur ces formules, l'échelle $\Lambda$ est une échelle de résolution invariante sous les dilatations, impossible à atteindre (pour cela, il faudrait une dilatation infinie à partir de toute échelle finie) et indépassable. Nous avons proposé de l'identifier, vers les très petites échelles, à l'échelle spatio-temporelle de Planck, $l_{p}=\left(\hbar G / c^{3}\right)^{1 / 2}=1.61605(10) \times 10^{-35} \mathrm{~m}$ et $t_{p}=l_{p} / c$, qui possèderait alors les propriétés physiques du point zéro tout en restant finie. Dans le cas macroscopique, on l'identifie à l'échelle de longueur cosmique donnée par l'inverse de la racine de la constante cosmologique ${ }^{8}$.

En quoi cette nouvelle loi de dilatation change-t'elle notre vision de l'espace-temps? À un certain niveau, elle implique une complication du fait de la nécessité d'introduire une cinquième dimension. Ainsi la métrique d'échelle s'écrit-elle à deux variables :

$$
d \sigma^{2}=\mathrm{d} \delta^{2}-(\mathrm{d} \ln \mathcal{L})^{2} / C_{0}^{2}, \text { avec } \mathbb{C}_{0}=\ln \left(\frac{\lambda_{0}}{V}\right) .
$$

L'invariant $d \sigma$ définit un «djinn propre », ce qui signifie que, bien que la dimension fractale effective soit devenue variable $\left(D^{\prime}=1+\delta^{\prime}\right.$ suivant $(9 b)$ ), la dimension fractale dans le repère propre (entrâné avec le système considéré) est restée constante.

Mais on peut également remarquer que la dimension fractale tend maintenant vers l'infini quand l'intervalle de résolution tend vers l'échelle de Planck. En allant à des résolutions de plus en plus petites, la dimension

8. Nottale, 1993 et 1996. 
fractale passera donc successivement par les valeurs 2,3 , 4, ce qui permettrait de couvrir une surface, puis l'espace, puis l'espace-temps à l'aide d'une unique coordonnée. Il est donc possible de définir un espace-djinn minkowskien nécessitant, dans des repères fractals adéquats, seulement deux dimensions aux très petites échelles. En allant vers les grandes résolutions, l'espace-temps-djinn voit sa cinquième dimension varier de moins en moins vite pour devenir presque constante aux échelles actuellement accessibles par les accélérateurs ${ }^{9}$, et finalement s'évanouir au-delà de l'échelle de Compton du système considéré (qui donne la transition fractal-non fractal dans le référentiel de repos), voici maintenant l'espace-temps minkowskien classique.

La relativité d'échelle restreinte et certaines de ses conséquences ont été développées plus à fond en ce qui concerne la mécanique relativiste d'échelle, la définition de nouveaux invariants et les applications en physique des hautes énergies ${ }^{10}$, ainsi que son lien avec les théories de supercordes ${ }^{11}$. Une représentation pleinement covariante d'échelle, et où mouvement et échelles soient traitées sur le même plan, reste cependant à construire.

4.3. Relativité d'échelle généralisée: transformations d'échelle nonlinéaires, champ d'échelle, nouvelle interprétation de l'invariance de jauge et $d u$ champ électromagnétique (couplage échelle-mouvement), relation masse-charge...

Ce vaste champ d'étude commence à peine à être abordé. C'est celui du passage à des transformations d'échelle non-linéaires et à la dynamique d'échelle, mais aussi de la prise en compte de résolutions qui dépendraient elles-mêmes des variables d'espace et de temps. Le premier volet mène au concept nouveau de champ d'échelle (qui correspond à une distorsion dans l'espace des échelles par rapport aux lois autosimilaires usuelles ${ }^{12}$ ); le second volet conduit à une nouvelle interprétation de l'invariance de jauge qui fournit des relations générales entre échelles de masse et constantes de couplage (charges généralisées) en physique des particules ${ }^{13}$. L'une de ces relations permet, en particulier, de prédire théoriquement la masse de l'électron (considérée dans cette approche comme essentiellement d'origine électromagnétique), en fonction de sa charge.

\footnotetext{
9. Nottale, 1996, fig. 4.

10. Nottale, 1992, 1993 et 1996.

11. CAstro, 1997.

12. Nottale, 1997 a et 1997 b.

13. Nottale, 1996.
} 


\section{5. - DYNAMIQUE INDUITE}

Ce domaine sera plus détaillé techniquement dans mon second article (voir infra, p. 93-116 dans ce même numéro). Il s'agit d'étudier les effets induits par les structures internes en échelle sur les lois du mouvement, c'est-à-dire sur l'espace-temps. On trouve que ces effets se couplent effectivement aux déplacements et impliquent des dédoublements de variables ainsi que l'apparition de termes nouveaux dans les équations différentielles. Cette description conduit à des lois indéterministes de type quantique (qui s'expriment en terme d'amplitudes de probabilité complexes, solutions d'équations de type Schrödinger) en deçà de l'échelle de transition fractal-non fractal, qu'on identifie à l'échelle d'Einstein-de Broglie (pour la particule libre).

\subsection{Effets induits par la non-différentiabilité}

Trois effets au minimum sont attendus dans la description des géodésiques d'un espace(-temps) non-différentiable :

\subsubsection{Infinité de géodésiques}

La multiplication à l'infini du nombre de géodésiques virtuelles dans un espace-temps fractal conduit à adopter une approche de type fluide et à définir un champ de vitesses $v(x(t), t)$ sur ce fluide de géodésiques.

\subsubsection{Brisure de symétrie $(d t \leftrightarrow-d t)$}

La nouvelle définition de la vitesse comme fonction explicite du temps et de l'élément différentiel (\$2) a une conséquence essentielle. Il n’y a pas une définition, mais deux,

$v_{+}(t, d t)=[x(t+d t)-x(t)] / d t$ et $v_{-}(t, d t)=[x(t)-x(t-d t)] / d t, \quad(12)$ qui se transforment de l'une en l'autre par la réflexion $d t \leftrightarrow-d t$. Ces deux vitesses n'ont a priori aucune raison d'être égales, non plus que leur moyenne (prise sur l'ensemble des géodésiques en un point donné). C'est ce dédoublement des vitesses, spécifique de la non-différentiabilité de l'espace-temps (qui va donc au-delà de son caractère fractal), que nous décrivons par la définition d'une vitesse complexe, et qui, dans cette interprétation, serait à l'origine du caractère complexe de la fonction d'onde en mécanique quantique.

\subsubsection{Fluctuations fractales}

Chaque géodésique est elle-même fractale, ce qui implique l'intervention de termes nouveaux dans les équations différentielles du mouvement 
moyen. C'est à ce niveau qu'on injecte les lois d'échelle déduites du principe de relativité $(\$ 4)$. Suivant le niveau considéré pour ces lois d'échelle, plusieurs niveaux de la dynamique induite sont donc à considérer :

(i) Relativité d'échelle galiléenne, dimension fractale $D=2$ (4.1)

Ce cas va correspondre au comportement quantique standard. Dans les situations les plus simples, on obtient, dans l'hypothèse où l'espace est fractal, l'équation de Schrödinger, et dans celle où l'espace-temps est fractal (qui correspond au passage à des vitesses et à des échelles relativistes du point de vue du mouvement), celle de Klein-Gordon.

(ii) $D \neq 2(4.1)$

On obtient, dans ce cas, en première approximation, une équation de Schrödinger généralisée explicitement dépendante d'échelle. Ainsi la dimension $D=2$ s'établit comme une dimension critique qui rend cachée la symétrie d'échelle (elle réapparaît indirectement dans les relations de Heisenberg). Cette situation mériterait d'être développée plus avant, en utilisant en particulier les méthodes d'intégro-différentiation fractionnaire.

(iii) $D$ variable (4.2 et 4.3 )

L'étude des effets induits pour une dimension variable, ou mieux qui devient la composante d'un vecteur comme en relativités restreinte et générale d'échelle («djinn »), n'a pour le moment été qu'ébauchée ${ }^{14}$, et seulement sous forme perturbative de «corrections relativistes d'échelle ». On s'attend à ce que ces effets se traduisent par des écarts à la mécanique quantique standard aux très hautes énergies atteintes par les futurs accélérateurs tel le LHC (Large Hadron Collider).

\subsubsection{Autres effets: autres brisures de symétrie, terme supplémentaire dans l'accélération, violation de CP}

Les équations de Pauli et Dirac restent à construire à partir d'un processus généralisé, une conjecture possible étant qu'elles résultent d'une brisure de parité différentielle, $d x \leftrightarrow-d x$. En ce qui concerne les accélérations, un terme supplémentaire pourrait devoir être introduit, puisque le dédoublement lié à la vitesse ne rend pas compte de tous ses aspects. Par ailleurs, l'irréversibilité locale qui mène à ce dédoublement des vitesses est supposée annulée une fois les vitesses avant et arrière combinées en une vitesse complexe : une voie de recherche consistera à étudier si un résidu de cette irréversibilité ne peut rendre compte de la violation de $\mathrm{CP}$ observée dans certaines expériences rares de physique des particules. Une autre possibilité de développement consisterait à abandonner la différentiabilité de l'espace des échelles lui-même, ce qui impliquerait de construire une mécanique quantique d'échelle, puis d'en rechercher les effets induits.

14. Nottale, 1996. 
5.2. Construction d'un opérateur de dérivation prenant en compte ces effets induits

Une fois les différents effets de la non-différentiabilité et de la fractalité décrits dans les déplacements élémentaires, on analyse leurs conséquences sur la variation des différentes grandeurs physiques dans ces déplacements. Ces conséquences sont décrites sous forme de termes supplémentaires aux opérateurs de dérivation ordinaire («dérivation covariante »).

\section{3. Équations d'Euler-Lagrange complexes, équation d'Hamilton-Jacobi}

Finalement les équations fondamentales de la physique sont réécrites en prenant en compte ces contributions nouvelles, en tentant de le faire à un niveau fondamental (principe de moindre action, équations de Lagrange).

5.4. Équations de Schrödinger, Klein-Gordon, Pauli, Dirac et généralisations via (4.1, $D \neq 2)$, (4.2), (4.3), (5.1.4)

On démontre ainsi certaines des équations fondamentales de la mécanique quantique, tout en élargissant leur domaine d'application ${ }^{15}$ et en en permettant des généralisations.

6. — DOMAINES D'APPLICATIONS

Ce point ne sera pas développé ici, car le présent article s'intéresse essentiellement à la structure des développements théoriques réalisés et envisageables. Il faut cependant rappeler pour mémoire que des applications ont été proposées dans plusieurs domaines ${ }^{16}$ : hautes énergies, formation de structures, cosmologie, voire à d'autres sciences (voir infra, p. 93116 dans ce même numéro).

Laurent NotTale ${ }^{17}$

(novembre 1999).

15. Nottale, 1997a.

16. Nottale, 1993, 1996 et 1997a.

17. J'exprime toute ma reconnaissance à Charles Alunni et à Éric Brian pour leur invitation à apporter une contribution à ce volume, ainsi qu'à Joël MERKER pour sa revue critique de mon ouvrage La Relativité dans tous ses états (1998) dans « Deux infinis cousus main », Revue de synthèse, 4e S., 1, janv.-mars 1999, p. 165-174. 


\section{LISTE DES RÉFÉRENCES}

CASTRo (Carlos), 1997, « String theory, scale relativity and the generalized uncertainty principle », Foundations of Physic Letters, vol. X, 3, p. 273-293.

EINSTEIN (Albert), 1916, « The foundation of the general theory of relativity », Annalen der Physik, vol. XLIX, p. 769, repr. in Albert EInsteIn, Hendrik A. Lorentz, Hermann Weyl et Hermann Minkowski, The Principle of relativity, trad. angl., New York, Dover publications, 1952, p. 109-164.

El Naschie (Mohamed S.), 1995, in Mohamed S. El Naschie, Otto E. Rössler et Ilya PRIGOGINe, éd., Quantum mechanics, diffusion and chaotic fractals, Oxford, Pergamon, p. 93, 185 et 191.

Mandelbrot (Benoît), 1975, Les Objets fractals, Paris, Flammarion.

Mandelbrot (B.), 1982, The Fractal Geometry of nature, San Francisco, CA, Freeman.

NotTALE (Laurent), 1992, «The theory of scale relativity », International Journal of Modern Physics, A7, p. 4899-4936.

Nottale (L.), 1993, Fractal space-time and microphysics. Towards a theory of scale relativity, Londres, World Scientific.

Nottale (L.), 1996, « Scale relativity and fractal space-time. Applications to quantum physics, cosmology and chaotic systems », Chaos, Solitons and Fractals, vol. VII, p. 877.

NotTale (L.), 1997a, « Scale relativity and quantization of the universe. I. Theoretical framework», Astronomy and Astrophysics, 327, p. 867.

Nottale (L.), 1997b, « Scale relativity », in Bérangère Dubrulle, François Graner et Didier Sornette, éd., Scale invariance and beyond, Proceedings of Les Houches school, Paris/Berlin, EDP Sciences/Springer, p. 249.

Nottale (L.), 1998, La Relativité dans tous ses états. Au-delà de l'espace-temps, Paris, Hachette Littératures.

Nottale (Laurent) et Schneider (Jean), 1984, «Fractals and non standard analysis », Journal of Mathematical Physics, vol. XXV, p. 1296-1300.

ORD (Garnet), 1983, «Fractal space-time. A geometric analogue of relativistic quantum mechanics », Journal of Physics. A : Mathematical and General, vol. XVI, p. $1869-1884$.

PoINCARÉ (Henri), 1905, Comptes rendus hebdomadaires des séances de l'Académie des sciences, Paris, 5 juin. 